\title{
Evaluation of a biochemical process to minimize snoring with ambroxol \& bromhexine in comparison with their performance in non OSA Bangladeshi patients
}

\author{
Sujit Biswas ${ }^{1}$, Md. Shah Aziz ${ }^{1}$, Sadique Mohammad Arif ${ }^{1}$, Md. Sohel Rana ${ }^{1}$ \\ '(Pharmacy Department, Jahangirnagar University, Dhaka, Bangladesh)
}

\begin{abstract}
Snoring is an important sign of sleep-related breathing disorders (SRBD), of the upper airway resistance syndrome (UARS), and of the obstructive sleep apnea (OSA). Snoring is a symptom of nasal obstruction and is associated with cardiovascular diseases. Roommates or bed partners are also suffered by snorers. In-house monitoring of snoring is very useful for evaluation by bed partner or roommate as well as polysomnography in sleeping centre. Process to evaluate and monitoring snoring available in the country are mechanical process. Our objective is to evaluate a biochemical process to minimize snoring with two mucolytic expectorants Ambroxol \& Bromhexine and compare their performance in non OSA Bangladeshi patients.32 snorers with no other respiratory disease like asthma, bronchitis etc. and who has ESS score 8-16 and SSS score 2-4,were taken from a Sleep Medicine Centre of Japan Bangladesh Friendship Hospital, 15 students from Bangabandhu Sheikh Mujibur Rahman Hall, Jahangirnagar University and another 15 from University of Development Alternative(UODA). The procedure and scoring were performed by help of roommates and bed partners. Snoring was measured on the basis of Frequency, Loudness, Snoring Index and day time sleepiness using Epworth Sleepiness Scale (ESS). A portable respiratory recording device (PRRD )was used to determine loudness, index and frequency of patient at home and snoring data was also collected from sleep medicine centre where polysomnogram was used. snoring severity objectively were also measured by using Snoring Severity Scale (SSS). excellent snoring suppressing effect was observed in both groups after treating separately with Ambroxol and Bromhexine $30 \mathrm{mg}$ per night. Snoring Frequency, Loudness, Severity, Index and ESS score was reduced markedly in compared to placebo group.
\end{abstract}

Keywords: polysomnogram, syndrome, mucolytic, Snoring, expectorant, OSA

\section{INTRODUCTION}

Snoring is a sign of abnormality. Snoring is a respiratory sound (or noise) which originates during sleep, and so can be nocturnal or diurnal. It is a typical inspiratory sound, even though a small expiratory component can be heard or recorded (especially in OSAS patients) with different spectral features. We must distinguish it from all the other sounds (noises) which can be heard, recorded and analyzed during sleep (sleep sounds syndrome, (SSS). The definition of snoring should also be related to the type of measure employed for sound and noise signals [1]. The American Sleep Disorders Association (ASDA) defined snoring as "Loud upper airways breathing, without apnea or hypoventilation, caused by vibrations of the pharyngeal tissues. It can be classified as mild, moderate and severe on the basis of frequency, body position, and disturbance for other people (spouse, bed partner)".

Moreover, ASDA defined primary snoring as snoring without sleep disruption and absence of complaint of insomnia or excessive daytime sleepiness [2]. Only recently has it been possible to show that obstructions which may be regarded as the cause of the snoring are formed especially by the drying out of the mucous membranes or additional mucous areas with micro fissures due to the deposit of tough mucus etc. When we are asleep, the area at the back of the throat sometimes narrows. The same amount of air passing through this smaller opening can cause the tissues surrounding the opening to vibrate, which in turn can cause the sounds of snoring. Different people who snore have different reasons for the narrowing. In most people, the reason for snoring is not known. Some potential causes (other than sleep apnea) include Being overweight, which leads to excessive neck tissue that puts pressure on the airways, last month of pregnancy, nasal congestion from colds or allergies, especially if it lasts a long time, swelling of the muscular part of the roof of the mouth (soft palate) or uvula, the piece of tissue that hangs down in the back of the mouth, swollen adenoids and tonsils that block the airways, use of sleeping pills, antihistamines, or alcohol at bedtime, drying out tissue of airways and larynx and reduction of muscle tone of soft palate or uvula etc.

A variety of factors can lead to snoring including your mouth anatomy, alcohol consumption, Nasal problems like chronic nasal congestion or a crooked partition between your nostrils and sleep apnea .Snoring leads to alterations that can reduce the life expectancy of the people afflicted by it. The most dangerous consequences appear to involve the cardiovascular apparatus. Snoring can also be a symptom of sleep apnea, a 
potentially life-threatening condition. While snoring is caused by narrow airways, sleep apnea is a true breathing obstruction, which requires the sleeper to awaken to begin breathing again. A person with sleep apnea wakes up many times a night to regain breathing, but usually remembers nothing at all about the awakenings. Snoring is a common symptom of sleep apnea, but snoring by itself does not involve the cessation of breathing. The most common type of sleep apnea is obstructive sleep apnea. During sleep, enough air cannot flow into your lungs through your mouth and nose even though you try to breathe. When this happens, the amount of oxygen in your blood may drop. Normal breaths then start again with a loud snort or choking sound.

There are various ways to alleviate this snoring problem such as non surgical (Behavioral changes, Dental devices, Nasal devices and medications, Nasal CPAP etc.) and surgical (deviated septum, nasal polyps, ulvectomy, LAUP and floppy plate etc.)

Although numerous investigations have been made to prevent snoring, they have not yet led to the desired success. Some of them are biochemical and some of them are mechanical even surgical also. The agents who used to treat snoring were composed on the foundation of chemo-therapeutics or antibiotics, corticoids or antihistaminics. But these agents have been found not to be active enough to prevent snoring over long periods, or they led, when taken over the long term, to damage to the nasal and pharyngeal mucous membrane. This applies also to the tests made earlier using etheric oils such as for example menthol, chamomile, eucalyptus oil etc., in higher concentration.

This bio chemical approach to fight against snoring by two expectorant Ambroxol and Bromhexine that contains an active content of a secretolytically and/or secretoproductively active substance together with the usual mucous membrane-compatible carriers and/or thinning agents, and which is suitable for oral administration as well as introduction into the nose-pharyngeal cavities. This a patented invention in USA upon which no specific research work was done. Recently a local pharmaceutical company vigorously promoted this campaign and tried to establish this patented innovation without any clinical trial. Unfortunately doctors' community was motivated with this promotion without any clinical reference and started to support their product. At that time a mighty interest was felt to evaluate this biochemical process and compare anti-snoring activity of ambroxol and bromhexine with placebo.

\section{Materials and Equipment}

The following materials were used to perform the test among the candidates designed for working out. It is represented here by name along with relevant information regarding its contribution to the test. It is presented in below alphabetical order.

a. A portable respiratory recording device (PRRD) (Sibel Home-300; Sibel SA, Barcelona, Spain) - The objective assessment of snoring and position is carried out by PRRD at home

b. Polysomnogram which produces Polysomnography, is a complete recording of the biophysiological changes that occur during sleep. The PSG monitors body functions including brain (EEG), eye movements (EOG), muscle activity or skeletal muscle activation (EMG), heart Rhythm (ECG) and breathing function and respirations effort. But our main objective was to measure respiratory function particularly breathing disturbances snoring and its frequency, index and snoring intensity determination

c. Ambrox was taken as Ambroxol Syrup in which each $5 \mathrm{ml}$ of syrup contains $15 \mathrm{mg}$ Ambroxol

hydrochloride BP. Ambroxol is a proven, reliable and effective expectorant

$\mathrm{d}$. Bromhexine- Bromhexine is known for having outstanding expectorant properties. It is more effective than vasicine in stimulating secretion of fluid by the mucous glands of the respiratory tract, thereby improving its clearance function. Bromhexine is appreciated as a reliable and safe secretolytic expectorant for the effective treatment of cough with phlegm.

e. B complex syrup - used as placebo.

\section{Study design}

Using a table of random numbers, subjects were randomized to Ambroxol Hydrochloride group (group1), Bromhexine group (group-2) or placebo group (group-3). Before therapy the patients completed two questionnaires and underwent an overnight full polysomnography. Snoring was recorded on four consecutive nights. Informed consent was obtained from all patients. In placebo group (group-3), Patients were told that the placebo treatment might improve upper airway function in sleep and reduce snoring.

\section{Method}

We recruited 32 snorers from a Sleep Medicine Centre of Japan Bangladesh Friendship Hospital (JBFH) and 15 from Bangabandhu Sheikh Mujibur Rahman Hall and 15 from University of Development Alternative (UODA). We selected snorer who has no other respiratory disease like asthma, bronchitis etc. and who has ESS (Epworth Sleepiness Scale) score $<16$ and primary or mild level snorer except two, who has ESS $>16$. They were not considered in the result though they perform the clinical trial. Roommates or bed partners 
help us to determine ESS and Snoring Severity Scale (SSS) score. Finally, we were able to select only 6 female and 44 male patients to participate in this trial. Among 50 patients, 45 patients were agreed to comply with the test rules and regulations. Patients were randomized and divided into three groups. Of all final selected patients, 15 patients from JFBA were subjected to polysomnography sleep study due to unavailability of the polysomnogram in Bangabandhu Sheikh Mujibur Rahman Hall and UODA.

Snorers recruited from JBFH have initial sleep study at sleep centre using polysomnography. But snorer recruited from Bangabandhu Hall and University of Development Alternative had to undergo sleep study at hall using portable respiratory recording device (PRRD), SSS and ESS to know if they are OSA patient or not. Patients having high ESS score $(>16)$ and snore loudly more than $50 \%$ night in a week are suspected as OSA patient.

We measured Snoring Frequency (number of snoring per minute), Loudness (in dB, only those were recorded whose sound within 45-60 dB), Snoring Index (number of snoring per hour) and day time sleepiness. Using Epworth Sleepiness Scale (ESS) and proper co-operation of bed partner or roommates we determined the day time sleepiness of patients, which is an important parameter of severity of snoring and correlated with obstructive sleep apnea (OSA) [3]. We used portable respiratory recording device (PRRD), which is worldwide accepted device to monitor respiratory disturbance, to determine loudness, snoring index and snoring frequency of patients at home and collected snoring data which was modified utilizing specific software at sleep centre of JBFH.

We also measured subjectively loudness of snoring by a set of questioner to bed partner or roommates. That questioner was used by many researchers in their study. That questioner contains 3 questions \# how often do you snore? \# how long do you snore? \# how audible is your snoring (with the door shut)? For each question according to severity score was allotted $0,1,2 \& 3$.Total score more than 7 suspected as OSA patient and excluded from study. Patient group were taken SSS score within 4-7out of 9 [4].

\section{Group I (Ambroxol)}

After successfully completion of previous polysomnography sleep study at JBFH, patients were convinced to take part in the study with the prior approval of eye nose throat (ENT) surgeon who suggested them to go sleep centre. They (15 patients) had taken $5 \mathrm{ml}(30 \mathrm{mg})$ Ambroxol each night before sleep and continued four consecutive nights. Among 15 patients 1 did not complete regimen.

\section{Group II (Bromhexine)}

After completion of polysomnography sleep study at JBFH, 15 patients were participated in the study with the advice of ENT surgeon who referred them to go sleep centre. They had taken $18.75 \mathrm{ml}(30 \mathrm{mg})$ Bromhexine each night before sleep and continued four consecutive nights. Among 15 patients one was affected by dry cough which could produce sound disturbance during snoring recording. For this reason he was excluded from study

\section{Group III (Placebo)}

The placebo treatment consisted of vitamin B complex syrup given to patient and told them that they will feel better than previous stage. Four consecutive nights $10 \mathrm{ml}$ vitamin B complex syrup each night was taken by them. Though 15 patients completed the regimen, result of two patients was excluded due to failure of loading data from PERD to computer to record them for use.

\section{Result and Discussion}

Ambroxol is a mucolytic agent used in the treatment of respiratory disorders associated with viscid or excessive mucus. It is the active ingredient of Mucosolvan or Ambrox. Bromhexine is used nearly same purpose. It is active ingredients of Bisolvon (International) or Mucolyt (Local).In the case of the administration of Ambroxol \& Bromhexine, Syrup was preferred as dosage form and dose of active ingredient of $30 \mathrm{mg}(5 \mathrm{ml}$ Ambroxol \& $18.75 \mathrm{ml}$ Bromhexine). Tests using $30 \mathrm{mg}$ Ambroxol produced excellent long-term effects whereas Bromhexine produced moderate effects in suppressing snoring. Although it was possible to administer a capsule preparation in which containing $75 \mathrm{mg}$ of ambroxol has a micro encapsulated $75 \mathrm{mg}$ Ambroxol- $\mathrm{HCl}$, which is a sustained release dosage form and Bromhexine tablet $8 \mathrm{mg}$. We could not assess result with 60 or $40 \mathrm{mg}$ ambroxol or bromhexine to evaluate responses which are giving gradually good result or not. We should use international brand Mucosolvan as reference standard but due to unavailability of the brand in the local market we had to choose Ambrox as reference. Manufacturer of Ambrox is SQUARE pharmaceuticals which is also reputed and UK, MHRA approved pharmaceutical industry in Bangladesh and also qualified in FDA standard in perspective of cGMP practice. Bisolvon is international brand and patented generic which was used as reference for bromhexine.

This is due to the fact that each group uses different instruments and devices, and their own recording protocols and data analysis. Particularly patient group taken from Bangabandhu Hall and UODA did not undergo 
polysomnogram. But accuracy of patient is not questionable. Because patients with suspected sleep apnea/ hypopnoea syndrome, the setting of unattended respiratory monitoring (home or laboratory) influences neither the number of valid studies nor the results of respiratory

However most patients, prefer home studies [5]. Very few number of female participants in the trial (6) which may be happen due to socioeconomic condition of our country. Firstly: not only female but also male feel shy to say or confess about his or her snoring habits. Many people have snoring habits, but they are not very aware of bad side of or risk associated with snoring. Secondly: those who are aware of snoring very few of them agree to perform sleep study at any sleep centre which is not only tedious and time consuming but also very much expensive. Besides this, available mechanical treatment is also very expensive.

Total 50 subjects (44 male \& 6 female) with a mean age 39 years (range $22-56$ years) completed the trial period as divided into three groups with ambroxol, bromhexine and placebo. Due to unavoidable circumstances 9 patients were excluded. Randomization resulted in 14 subjects receiving ambroxol, 14 patients' bromhexine and 13 patients' placebos. Comparison of baseline data obtained from polysomnography at the sleep medicine centre and home utilizing PERD did not show significant difference (Table-3.1). These subjects were excluded from outcomes. To clinically investigate and compare capability of ambroxol and bromhexine to fight against snoring base line data of each group was compared to pooled data obtained from four night sleep study. These results are shown in Table 3.2.

Measurement of snoring using PERD has some limitations. This method provides an estimation of the total length of time spent snoring on the individual night of the recording [6]. Besides this many subjects do not snore in every night. And it is also recognized that duration, timing, loudness of the snoring may vary from night to night in case of individual snorer. Consequently, we can say PERD technology may assess severity of snoring poorly. In this monitoring system include loudness of snoring can be measured but within a range of 45$60 \mathrm{~dB}$. If out of this range snores a patient, the recorder cannot register it (Fig:3.6). For this reason we used a subjective approach to measure loudness of snoring. We used a questioner which was widely accepted. But problem of this method is interpersonal variation. It is suppose that we evaluated a snorer through questioner to his or her bed partner or roommate. The person own self (evaluator) can be deep sleepy or it may vary person to person evaluation.

The normal upper limit of ESS scores is 10 but in the present study 19 subject (Fig: 5 \& 10)was had base line ESS score of more than 10 indicating that these subjects apparently increases base line level of daytime sleepiness. The lower ESS scores reported with the ambroxol may reflect that these subjects experienced less disturbed sleep with this medicine although, as these subjects had normal baseline oxymetry, the clinical significance of these findings should be interpreted with great caution. It is very important high score of ESS response low. Baseline data from these patients of ambroxol group consisted of: age: $40 \pm 17$ yrs; BMI: $27.5 \pm 3.7$ kg.m ${ }^{-2}$; ESS: $12 \pm 4$; SF: $6.36 \pm 2.88$; SI: $298 \pm 168$ snores h-1; dB med: $52.25 \pm 6.78$ dB sound pressure level (SPL) and Snoring Severity Score (SSS) 3.08 \pm 0.79 . Bromhexine group consisted of: age: $36 \pm 14$ yrs; BMI: $24.3 \pm 4.6$ kg.m ${ }^{-2}$; ESS: $12 \pm 4$; SF $6.76 \pm 2.29$; SI: $286 \pm 171$ snores h-1; dB med: $52.92 \pm 5.28 \mathrm{~dB}$ sound pressure level (SPL) and Snoring Severity Score (SSS) 3.17 \pm 0 . 81. Placebo group consisted of: age: 42 \pm 9 yrs; BMI: $25.1 \pm 4.9$ kg.m ${ }^{-2}$; ESS: $11 \pm 3$; SF $6.83 \pm 2.23$; SI: $279 \pm 172$ snores h-1; dB med: $53.5 \pm 5.2 \mathrm{~dB}$ sound pressure level (SPL) and Snoring Severity Score (SSS) $3.23 \pm 0.59$. Significant differences were not found in the baseline data among ambroxol group, bromhexine and placebo group for the parameters in table 3.1. Pooled data from ambroxol patients consisted of: age: $40 \pm 17$ yrs; BMI: $27.5 \pm 3.7$ kg.m ${ }^{-2}$; ESS: $9 \pm 5$; SF: $4.58 \pm 3.46$; SI: $145 \pm 131$ snores h-1; dB med: $49.72 \pm 4.68 \mathrm{~dB}$ sound pressure level (SPL) and Snoring Severity Score (SSS) $1.24 \pm 0.96$. Pooled data from bromhexine patients consisted of: age: $36 \pm 14$ yrs; BMI: $24.3 \pm 4.6$ kg. $\mathrm{m}^{-2}$; ESS: $10 \pm 5$; SF: $5.62 \pm 3.49$; SI: $176 \pm 159$ snores h-1; dB med: $48.67 \pm 3.48$ dB sound pressure level (SPL), Snoring Severity Score (SSS) $1.34 \pm 0.89$. Significant variation did not found in placebo group. Pooled data from Placebo group consisted of: age: $42 \pm 9$ yrs; BMI: $25.1 \pm 4.9$ kg.m ${ }^{-2}$; ESS: $11 \pm 4$; SF $6.96 \pm 2.23$; SI: $257 \pm 152$ snores h-1; dB med: $52.5 \pm 4.6 \mathrm{~dB}$ sound pressure level (SPL) and Snoring Severity Score (SSS) 2.76 $\pm 0.97$

Only three patients response well during placebo controlled trial. That may happen due to either genuine placebo effect or having truly any snoring suppressing effect of Vitamin B-complex. This can also be due to changing sleeping positioning or changing alcohol or smoking habit. Particularly two of them stopped smoking during trial. This is one of our great limitations during study; we could not monitor well smoking of patients and other factors which can influence severity of snoring. We also could not justify effect of ambroxol and bromhexine on severe snorer with high ESS score.

Table 3.1: Base line data of snorers before treatment

\begin{tabular}{|l|l|l|l|}
\hline Parameter & Ambroxol & Bromhexine & Placebo \\
\hline Age & $40 \pm 17$ yrs & $36 \pm 14$ & $42 \pm 9$ yrs \\
\hline \multicolumn{2}{|c|}{ www.iosrjournals.org } \\
\hline \multicolumn{2}{|c|}{8 Page }
\end{tabular}


Evaluation of a biochemical process to minimize snoring with ambroxol \& bromhexine in comparison

\begin{tabular}{|l|l|l|l|}
\hline Body mass Index & $27.5 \pm 3.7 \mathrm{~kg} \cdot \mathrm{m}-2$ & $24.3 \pm 4.6 \mathrm{~kg} . \mathrm{m}-2$ & $25.1 \pm 4.9 \mathrm{~kg} . \mathrm{m}-2$ \\
\hline Limits of Sound intensity & $45-60 \mathrm{~dB}$ & $45-60 \mathrm{~dB}$ & $45-60 \mathrm{~dB}$ \\
\hline dB median & $52.25 \pm 6.78$ & $52.92 \pm 5.28$ & $53.5 \pm 5.2$ \\
\hline Snoring Index & $298 \pm 168$ & $286 \pm 171$ & $279 \pm 172$ \\
\hline Snoring Frequency & $6.36 \pm 2.88$ & $6.76 \pm 2.29$ & $6.83 \pm 2.23$ \\
\hline Snoring Severity Scale & $3.08 \pm 0.79$ & $3.17 \pm 0.81$ & $3.23 \pm 0.59$ \\
\hline Epworth Sleepiness Scale & $12 \pm 4$ & $12 \pm 4$ & $11 \pm 3$ \\
\hline
\end{tabular}

Table 3.2: Pooled data of snorers after treatment

\begin{tabular}{|l|l|l|l|}
\hline Parameter & Ambroxol & Bromhexine & Placebo \\
\hline Age & $40 \pm 7$ yrs & $36 \pm 14$ & $42 \pm 9$ yrs \\
\hline Body mass Index & $27.1 \pm 2.9 \mathrm{~kg} . \mathrm{m}-2$ & $24.3 \pm 4.6 \mathrm{~kg} . \mathrm{m}-2$ & $25.1 \pm 4.9 \mathrm{~kg} . \mathrm{m}-2$ \\
\hline Limits of Sound intensity & $45-60 \mathrm{~dB}$ & $45-60 \mathrm{~dB}$ & $45-60 \mathrm{~dB}$ \\
\hline dB median & $49.72 \pm 4.68$ & $48.67 \pm 3.48$ & $52.5 \pm 4.6$ \\
\hline Snoring Index & $145 \pm 131$ & $176 \pm 159$ & $257 \pm 152$ \\
\hline Snoring Frequency & $4.58 \pm 3.46$ & $5.62 \pm 3.49$ & $6.96 \pm 2.23$ \\
\hline Snoring Severity Scale & $1.24 \pm 0.96$ & $1.34 \pm 0.89$ & $2.76 \pm 0.97$ \\
\hline Epworth Sleepiness Scale & $9 \pm 5$ & $10 \pm 5$ & $11 \pm 4$ \\
\hline
\end{tabular}

The graph shown bellow implies that time between two snoring sound or respiratory disturbance sound reducing with administration of ambroxol hydrochloride which expressed through distance of two bold signals. Besides this severity in decibel also reducing. Graph upper and lower limit also minimized.

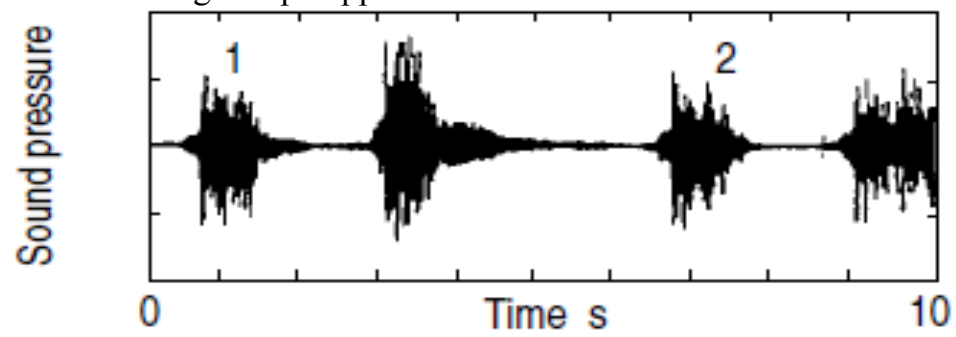

Fig 3.1: Severity and Intensity of snoring before treatment

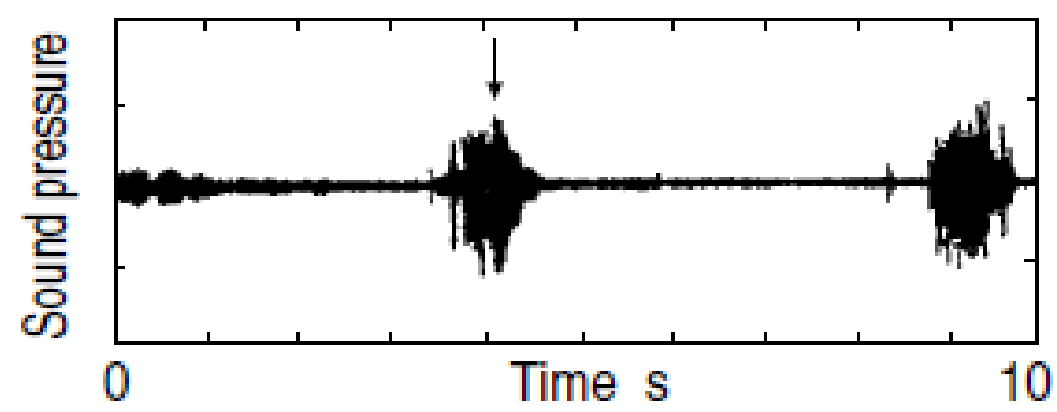

Fig 3.2: Severity and Intensity of snoring after treatment

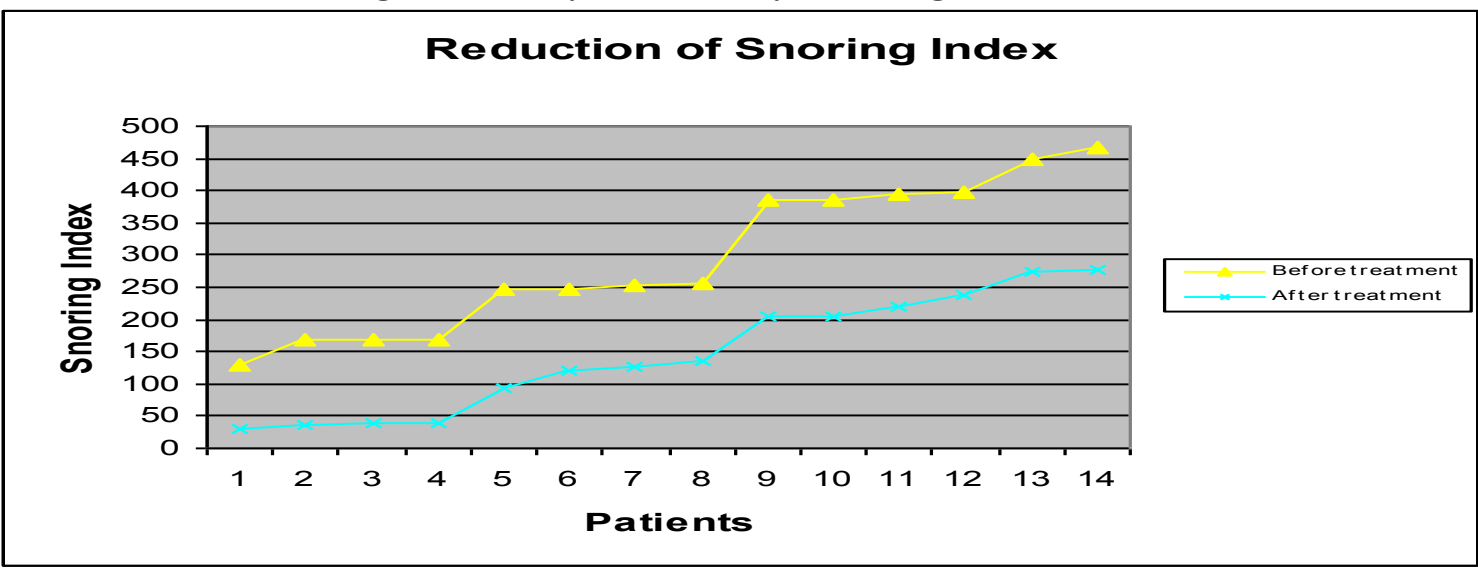

Fig 3.3: Pre and post treatment Snoring Index Comparison with ambroxol 


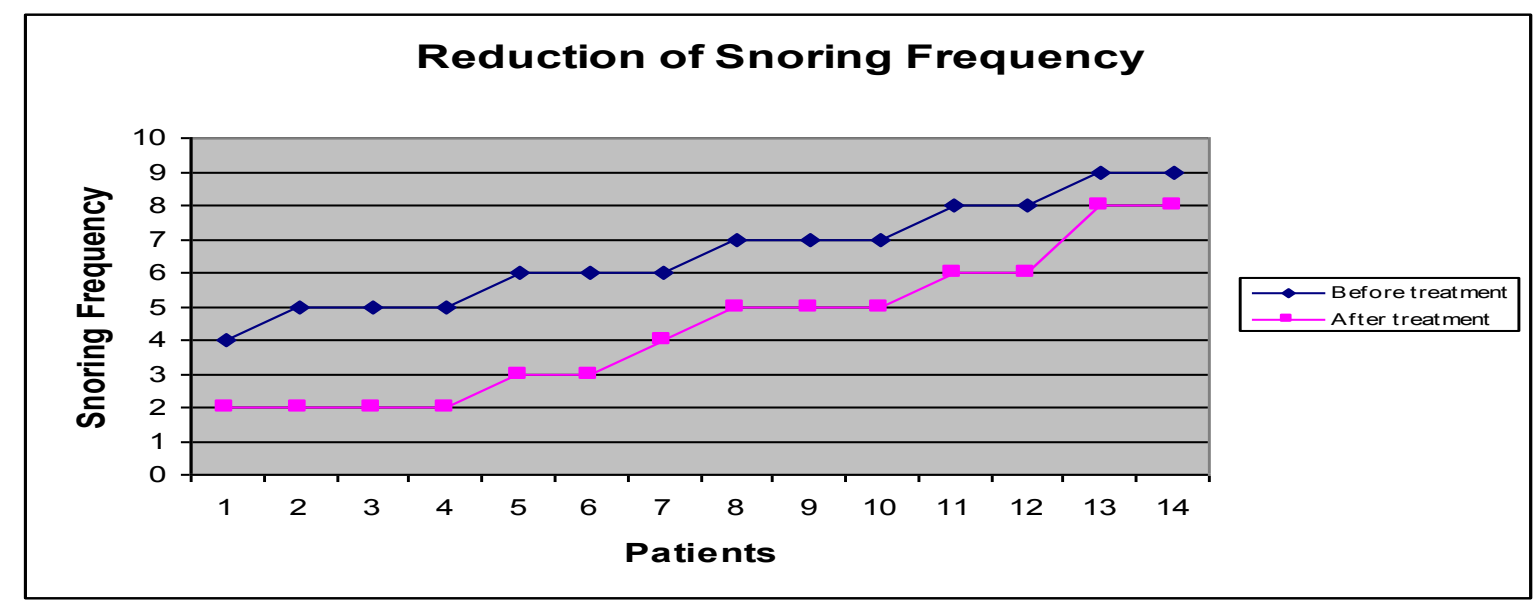

Fig 3.4: Pre and post treatment Snoring Frequency Comparison with ambroxol

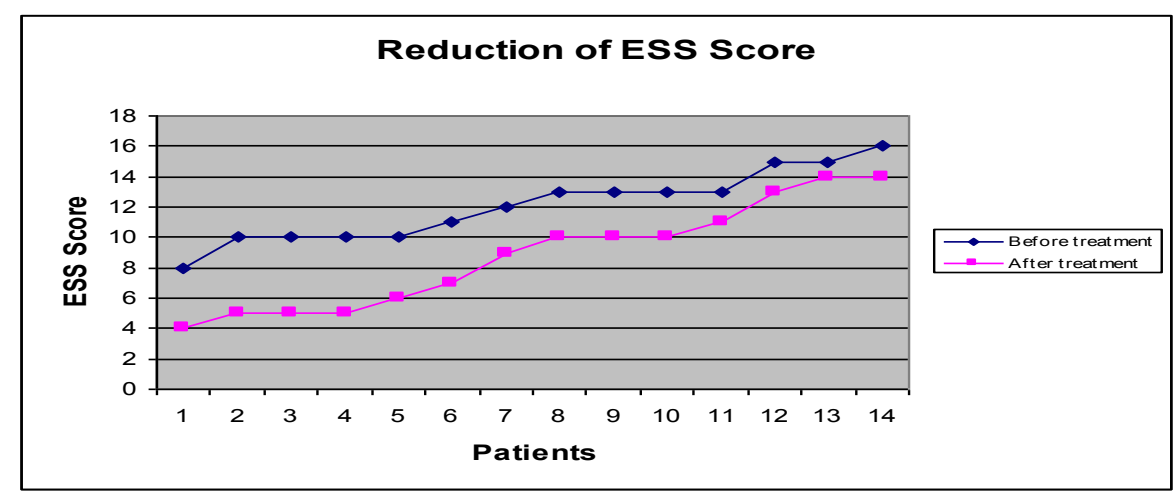

Fig 3.5: Pre and post treatment Epworth Sleepiness Scale Score Comparison with ambroxol

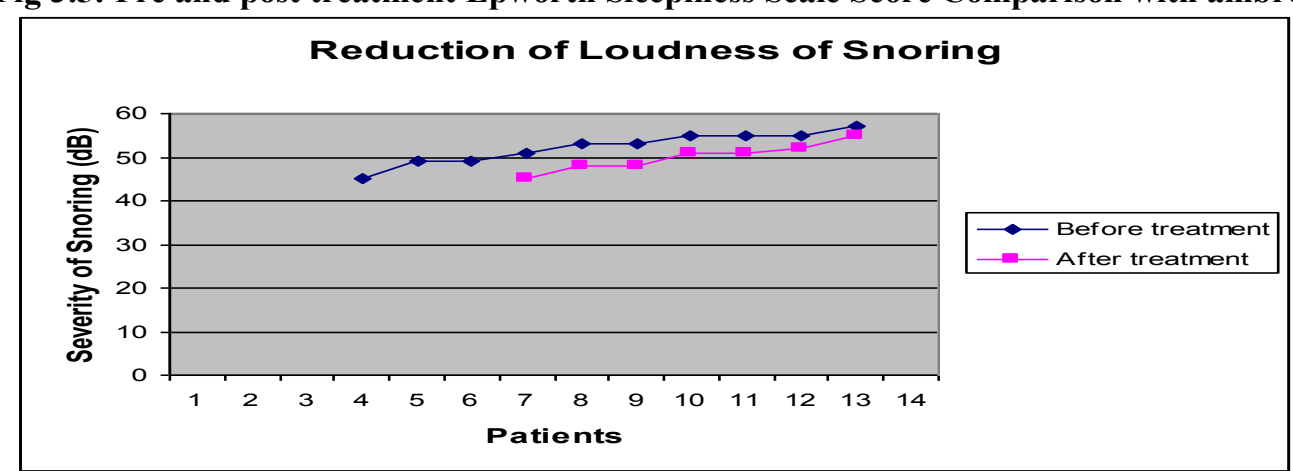

Fig 3.6: Pre and post treatment Loudness of Snoring (dB) Comparison with ambroxol

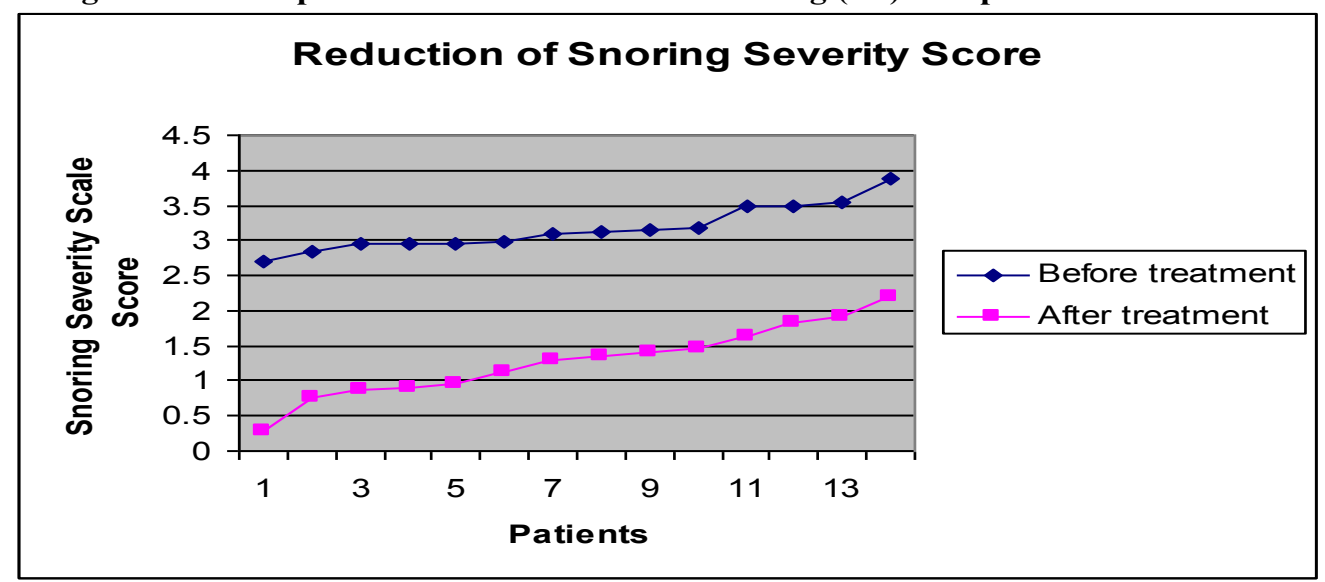

Fig 3.7: Pre and post treatment Severity of Snoring Comparison with ambroxol 


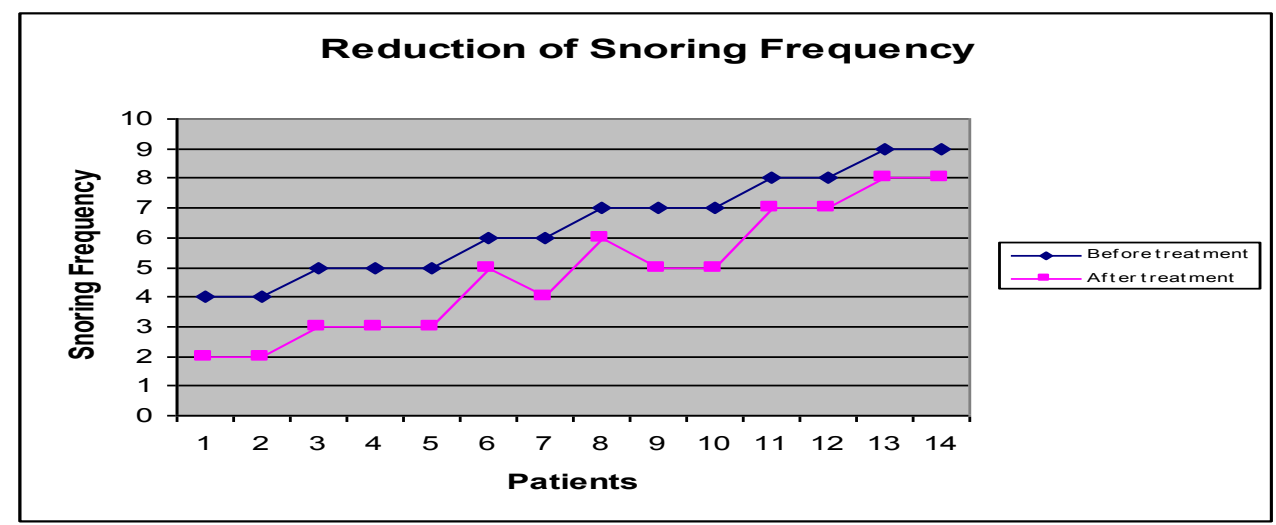

Fig 3.8: Pre and post treatment Snoring Frequency Comparison with bromhexine

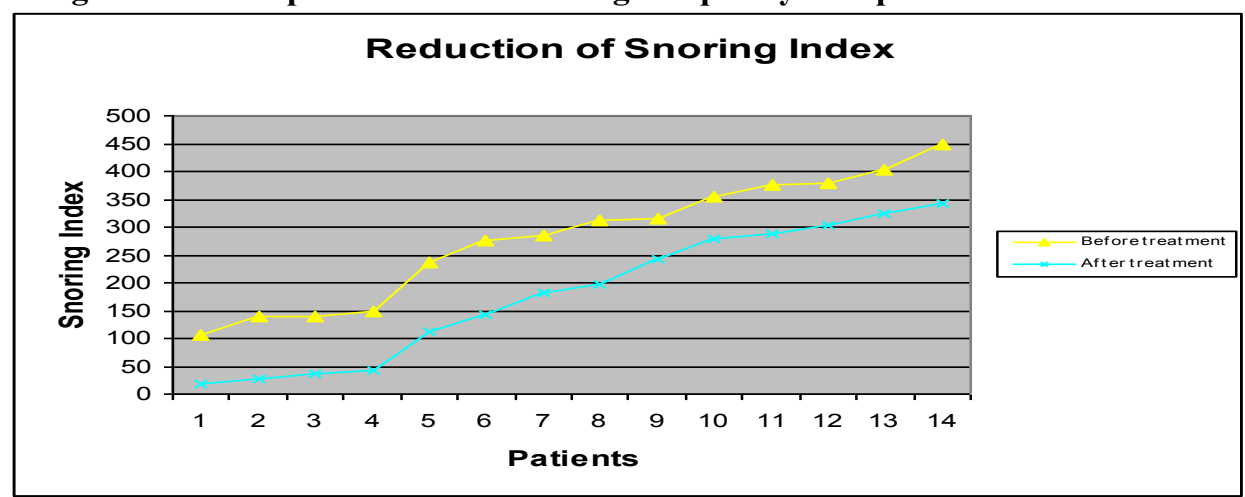

Fig 3.9: Pre and post treatment Snoring Index Comparison with bromhexine

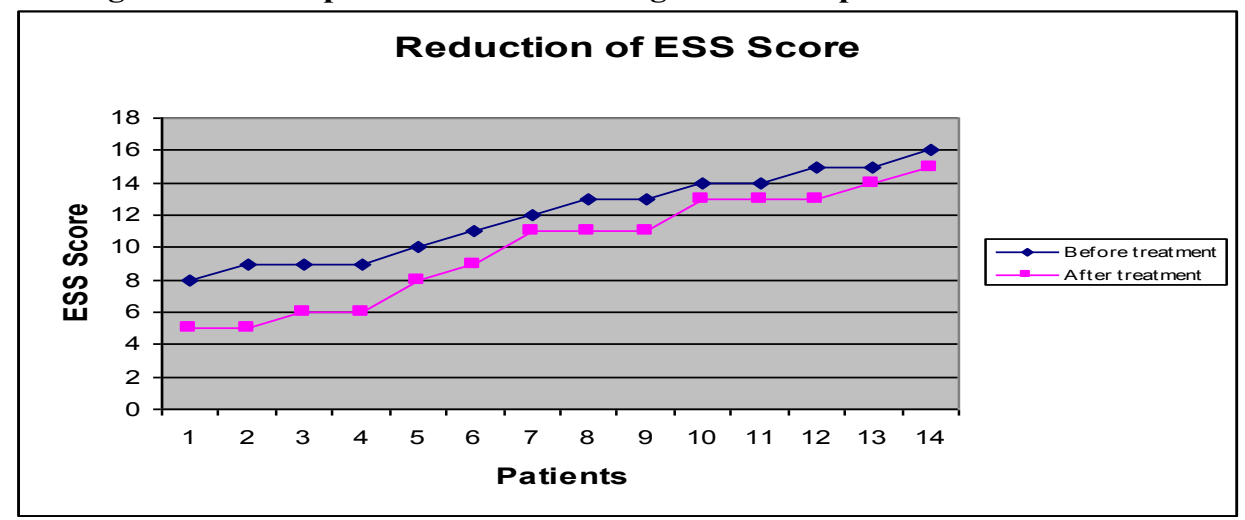

Fig 3.10: Pre and post treatment ESS score Comparison with bromhexine

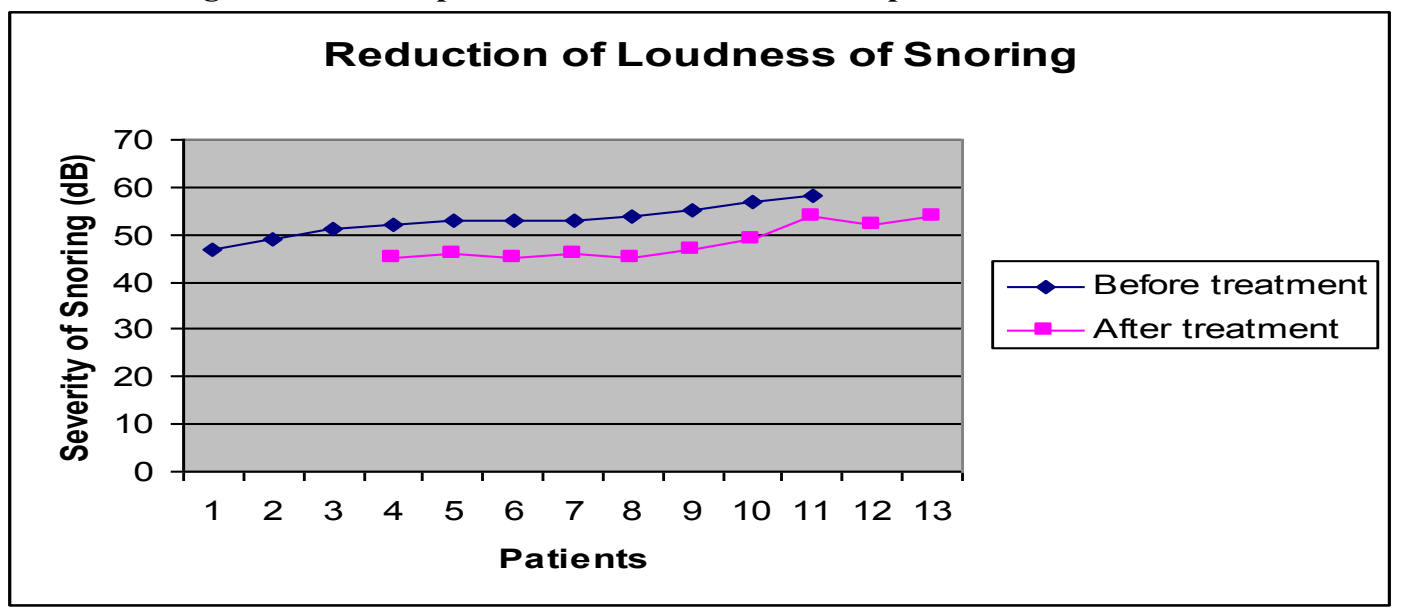

Fig 3.11: Pre and post treatment loudness of Snoring Comparison with bromhexine 


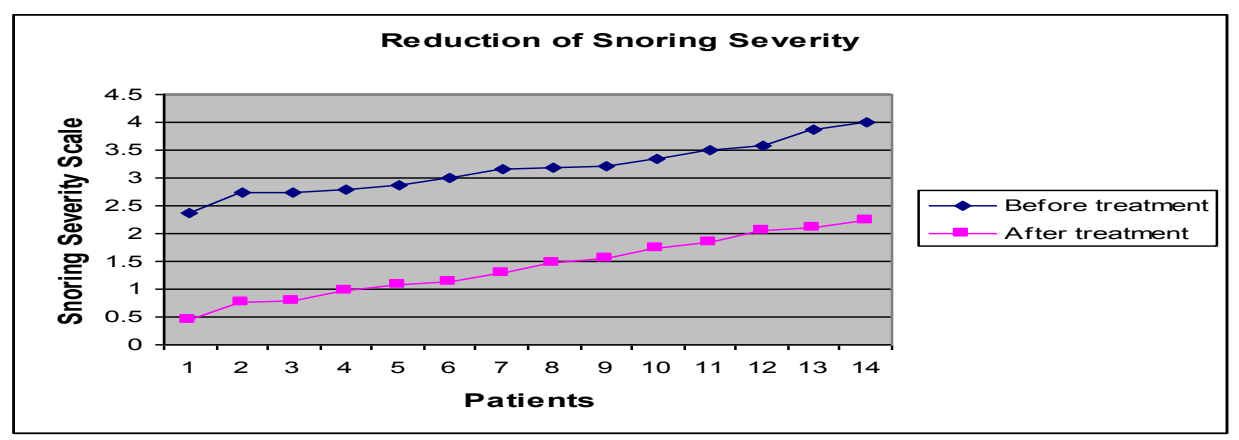

Fig 3.12: Pre and post treatment Severity of Snoring Comparison with bromhexine

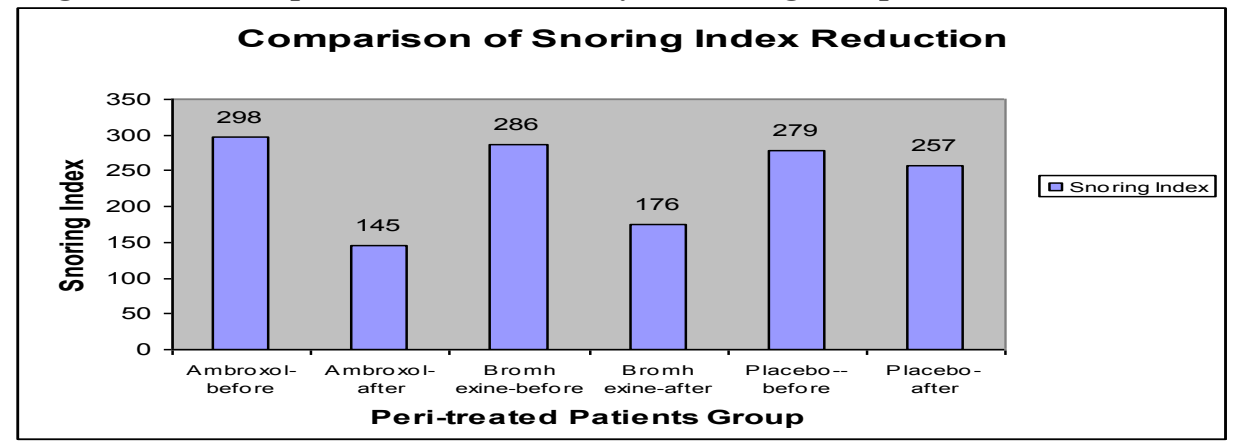

Fig 3.13 Pre and post treatment Snoring Index comparison

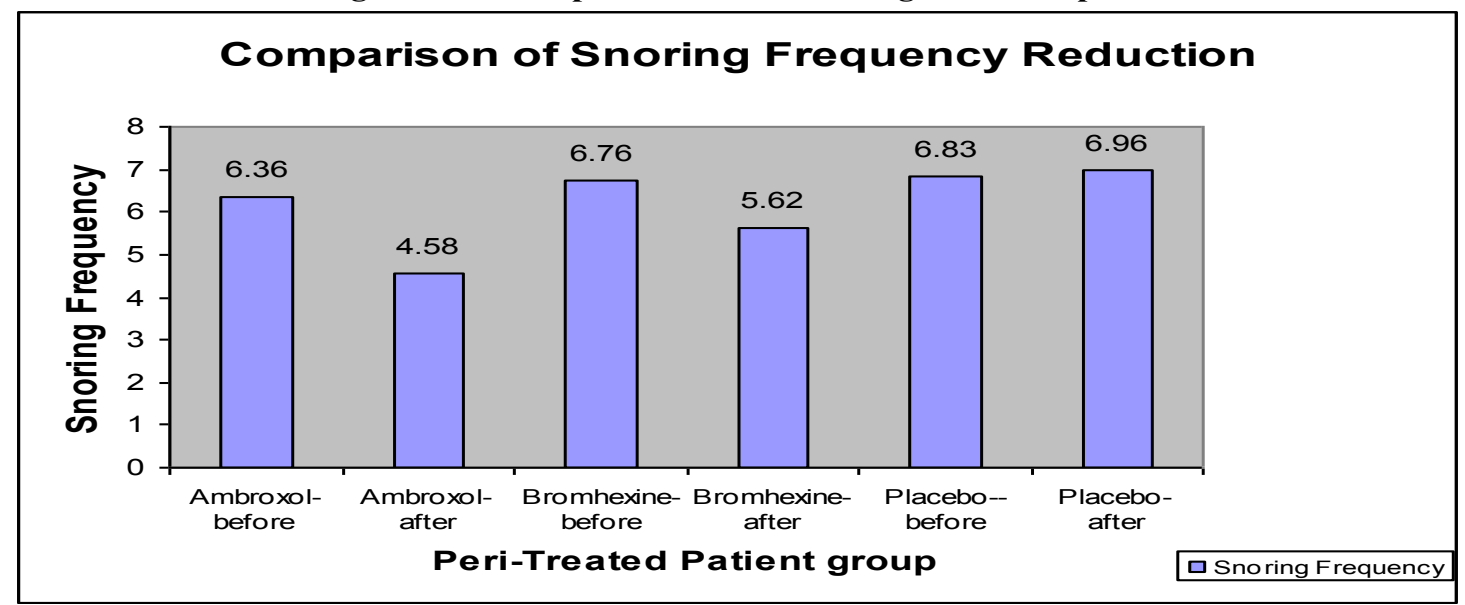

Fig 3.14 Pre and post treatment Snoring Frequency comparison

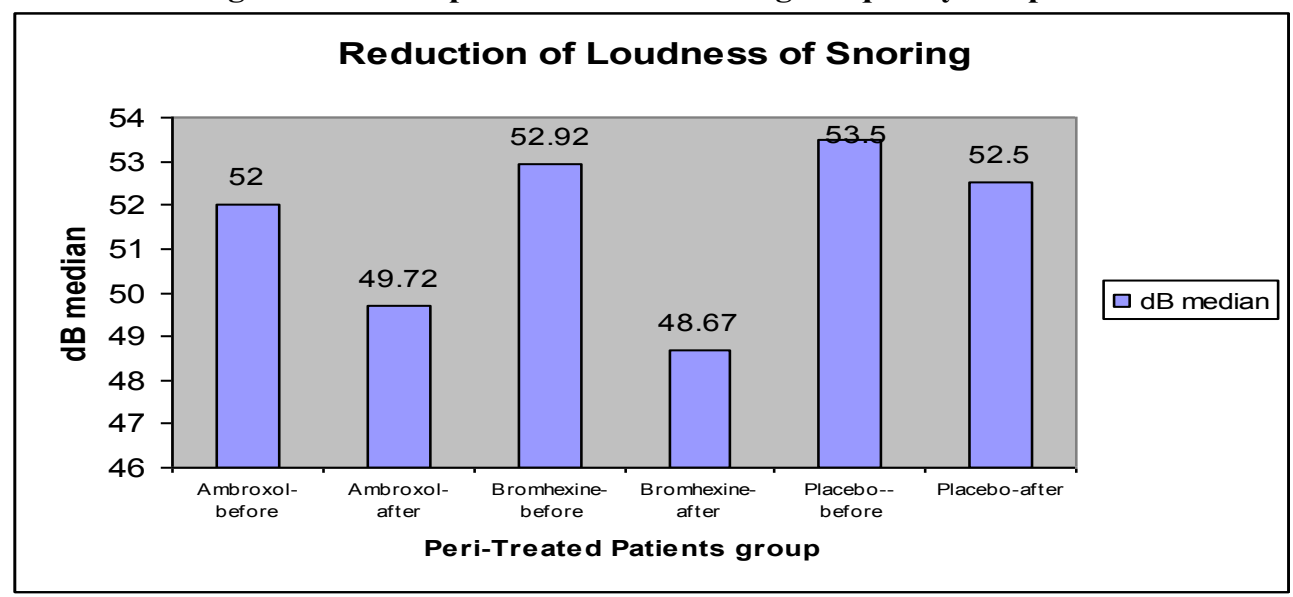

Fig 3.15 Pre and post treatment Loudness of Snoring comparison 


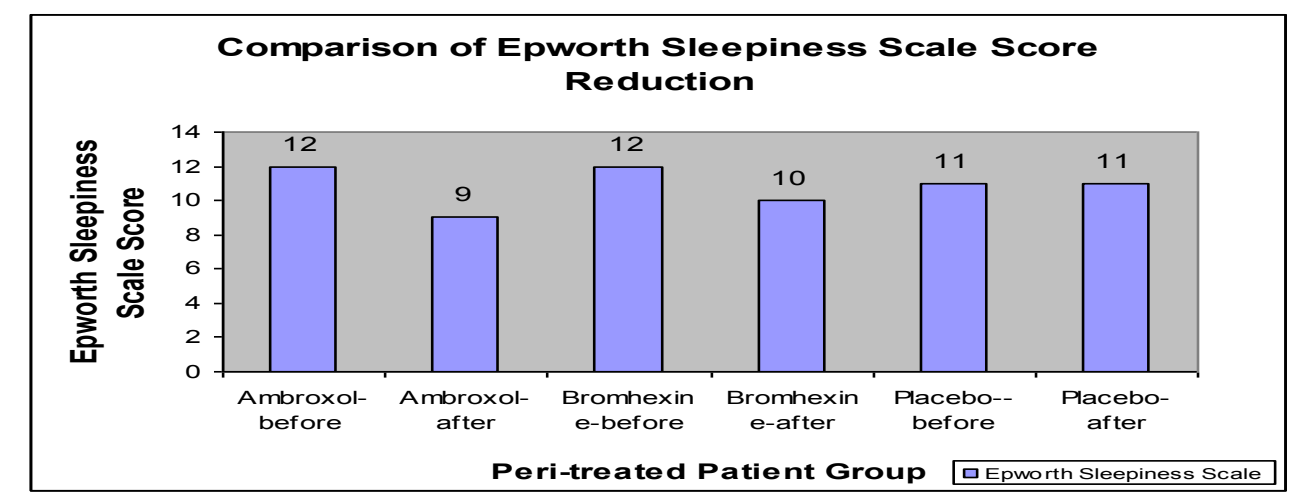

Fig 3.16 Pre and post treatment ESS score comparison

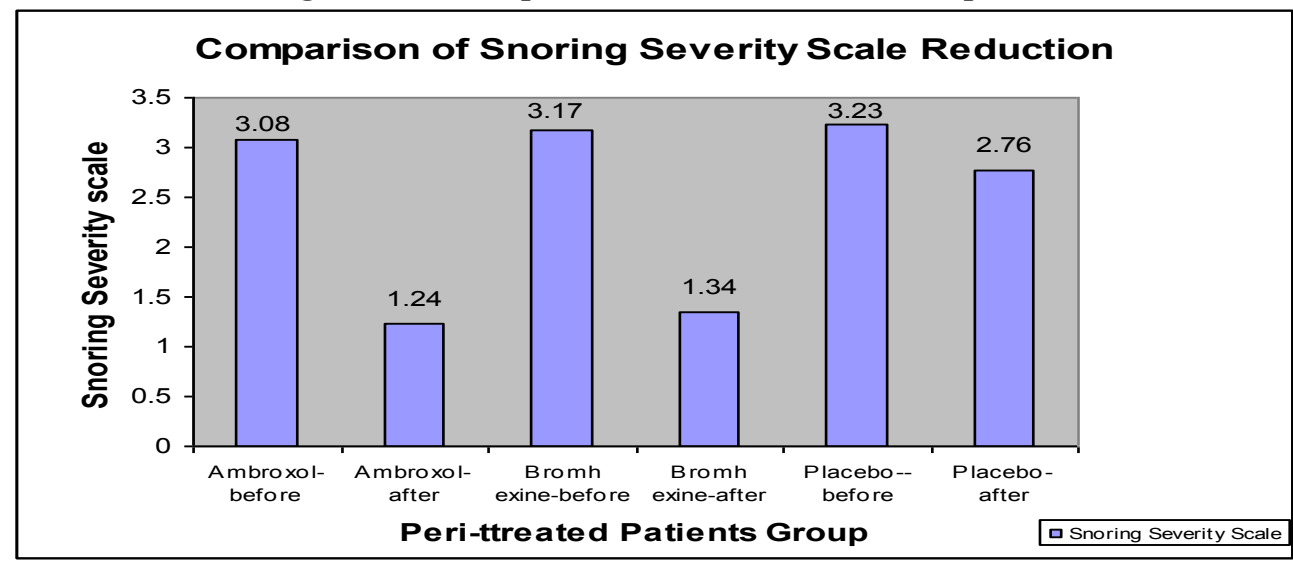

Fig 3.17 Pre and post treatment SSS score comparison

The oral anti-snoring agents Ambroxol or Bromhexine expedite the function of the mucous membrane glands of the respiratory tract with usual carriers or thinning agents. Further the study comprises a process for the use of inventive anti-snoring agent. Ambroxol and Bromhexine are especially advantageous to fight against snoring due to having a few unique properties. They are regulation and normalization of the mucus viscosity, reduction of mucus adhesion by the activation of endogenous surfactant properties, stimulation and improvement of theserous mucus production and secretion and activation and improvement of the operation of the mucoceliary function. The secretolytic drug absorbed by the nasal and pharyngeal mucous membrane causes a stimulation of the mucous membrane glands, whereby the result is a liquification of tough mucus which covers the mucous membrane, and it leads to a Stimulation of the mucus secretion, whereby the drying out of the mucous membranes and the formation of micro fissures therein are prevented. But to prevent snoring, muscle tone improvement is required. How does ambroxol improve muscle tone, is not clear yet or how does wet soft tissue increase muscle tone of soft palate and airways tissues, is not clear yet. Besides, this soft tissue dries out every day but a patient usually does not snore every day. So it may be questionable about the mechanism of action of ambroxol and bromhexine.

Better result of ambroxol than bromhexine can be explained with its structure activity relationship. From the structure we can see that ambroxol and bromhexine have almost similar structure except one hydroxyl group in the ring of ambroxol which definitely increase the affinity and intrinsic activity of ambroxol to $\beta_{2}$-receptor. Perhaps this hydroxyl group is responsible for contra-indication of ambroxol with antitussives where bromhexine is used. Besides this, bromhexine was lead compound of ambroxol and both of them are synthesized from vasicine alkaloid. Exception also exist, loudness of snoring was reduced with bromhexine group more than ambroxol group.

\section{Conclusion}

On the basis of the study result and discussion, it can be concluded that Ambroxol \& bromhexine both reduce severity of snoring and day time sleepiness in nonapnic snorer in comparison to placebo group. Ambroxol shows more efficacy than bromhexine in snoring suppressing effect. Further study can be conducted on apneic patient. Further investigation is required to observe response against $45 \& 60 \mathrm{mg}$ dose.Sleep study should be conducted in sleep centre instead of home to get more accurate result. How salivary secretion improves muscle tone and reduces snoring that is not clear yet. After discontinuation treatment with ambroxol \& bromhexine what happens that should also be observed in further study. 


\section{Acknowledgement}

This study was carried out among the students of Bangabandhu sheikh Mujibur Rahman Hall, Jahnagirnagar University, University of Development Alternatives. Japan Bangladesh Friendship Hospital in 2009. Our deepest gratitude goes to Dr. Md. Shohel Rana sir for his patience and guidance while we struggled through the periods of project work. His enthusiastic supervision and support inspired us remarkably to complete this work. We are most grateful to Dr. Monilal Aich, a famous ENT (Ear, Nose \& Throat) specialist of the country and Asst. Professor of DMCH (Dhaka Medical College Hospital), for his instruction and encouragement concerning this work. We are also grateful to Dr. Sharder A. Baki, Consultant of Sleep Medicine, Sleep Centre, Japan Bangladesh Friendship Hospital, for his proper co-operation.

We thank acquaintances in Popular Pharmaceuticals Ltd., batch mates and juniors in Bangabandhu Sheikh Mujibur Rahman Hall for their active participation in clinical trial. Finally, We owe the warmest gratitude to our family members for their everlasting support and encouragement during the years of studies.

\section{References}

[1] Dalmasso F, Benedetto G, Righini G and Spagnolo R, Snoring sound analysis and acoustic tube model of upper airway. Proc. of the 14th Int. Conf. on Lung Sounds, Winnipeg, 1989.

[2] Thorpy MJ, The international classification of sleep disorders: diagnostic and coding manual. Lawrence KS, ed. Allen Press Inc., USA pp. 1990, 195-197.

[3] Johns MW, A new method for measuring daytime sleepiness: the Epworth sleepiness scale. Sleep, 1991, 14: 540-5

[4] Lim PV and Curry AR, A new method for evaluating and reporting severity of snoring, Journal of Laringology and Otology, 1999, 336-340 (113)

[5] P. Lloberes, G. Sampol, G. Levy, D. Aristizabal, T. Sagales, M. De la Calzada, A. Roca, I. Can,and F. Morell, Influence of setting on unattended respiratory monitoring in the sleep apnoea/hypopnoea syndrome. Eur. Respir. J. 2001, 18: 530-534.

[6] Stradling JR and Crosby JH, Relation between systemic hypertension and sleep hypoxaemia or snoring: analysis in 748 men drawn from general practice. Br. Med. J. 1990, 300: 75-78. 